\title{
Sjögren's syndrome in patients with chronic idiopathic axonal polyneuropathy
}

\author{
G W van Dijk, N C Notermans, L Kater, A A Kruize, W H J P Linssen, J H J Wokke
}

\section{Departments of \\ Neurology \\ G W van Dijk \\ N C Notermans \\ J H J Wokke}

Rheumatology and Clinical Immunology,

University Hospital,

Utrecht, The

Netherlands

L Kater

A A Kruize

\section{Department of Neurology, St \\ Lucas-Andreas \\ Hospital, Amsterdam, \\ The Netherlands \\ W H J P Linssen \\ Correspondence to: \\ Dr GW van Dijk, \\ Department of \\ Neuromuscular Diseases, \\ University Hospital, Utrecht, \\ PO Box 85500, C03.236, \\ 3508 GA Utrecht, The \\ Netherlands.}

Received 21 January 1997 and in revised form 19 March 1997

Accepted 3 April 1997

\begin{abstract}
Objective-To assess the presence of symptoms and signs of Sjögren's syndrome in patients with otherwise idiopathic axonal polyneuropathy and to develop guidelines for the diagnostic approach with respect to Sjögren's syndrome in these patients.

Methods-Sixty five patients with axonal polyneuropathy in whom an aetiological diagnosis could not be made underwent (1) a standard interview focusing on ocular and oral sicca symptoms, (2) physical examination, (3) tests for objective assessment of keratoconjunctivitis sicca, (4) extensive serological investigations, and (5) a sublabial salivary gland biopsy.

Results-In forty nine patients a sublabial salivary gland (SSG) biopsy was performed, thereby completing the whole investigation for Sjögren's syndrome. Three of these 49 patients (all women) had an SSG biopsy specimen suggestive of Sjögren's syndrome, which, in combination with other symptoms and signs, led to a diagnosis of primary Sjögren's syndrome.

Conclusions-None of the three patients with primary Sjögren's syndrome had spontaneously complained about sicca symptoms and the clinical neurological picture of them did not differ from the other patients in the study. Therefore, in patients with chronic idiopathic axonal polyneuropathy, especially in women, a systematic investigation for Sjögren's syndrome should be done, because the presence of Sjögren's syndrome may have implications for treatment and justifies a clinical follow up on a regular base.
\end{abstract}

(F Neurol Neurosurg Psychiatry 1997;63:376-378)

Keywords: Sjögren’s syndrome; axonal; polyneuropathy

In about $15 \%$ of patients with a chronic axonal polyneuropathy a cause cannot be found despite extensive investigations. ${ }^{1}$ It is not clear whether all of these patients should undergo diagnostic tests for Sjögren's syndrome and if so, which tests. In Sjögren's syndrome different types of neuropathies can be recognised, such as distal symmetric neuropathy, mononeuropathy, sensory neuronopathy, and autonomic neuropathy. ${ }^{2}$ Polyneuropathy can be the first clinical manifestation of Sjögren's syndrome and it has been reported to precede sicca symptoms in $39 \%$ of patients. ${ }^{3}$ Diagnosing Sjögren's syndrome is important, as some patients run an unfavourable course and may benefit from immunosuppressive treatment. ${ }^{3}$ Furthermore, patients with primary Sjögren's syndrome have a 44 times higher relative risk of developing malignant lymphoma and a 50\% chance of developing a monoclonal gammopathy, which justifies a regular clinical follow up in these patients. ${ }^{4}$

We investigated the presence of features of Sjögren's syndrome in patients with chronic idiopathic axonal polyneuropathy (CIAP) by systematically testing these patients for ocular, oral, and systemic involvement. The aim of the study was to identify predicting factors for the presence of Sjögren's syndrome in patients with otherwise idiopathic axonal polyneuropathy and to develop guidelines for the diagnostic approach to Sjögren's syndrome in these patients.

\section{Methods}

In a prospective study between 1992 and 1995, 65 patients were diagnosed as having CIAP. All patients underwent a standard evaluation as described previously. ${ }^{5}$ No cause for the polyneuropathy was found in any of the patients (a raised erythrocyte sedimentation rate as the only abnormal finding was no exclusion criterion). Furthermore, all patients were investigated according to a standard protocol with respect to the presence of features of Sjögren's syndrome. A sublabial salivary gland (SSG) biopsy was performed after having obtained informed consent, thereby completing the clinical investigation for Sjögren's syndrome.

\section{PATIENT EVALUATION WITH RESPECT TO} SJÖGREN'S SYNDROME

The following investigations were performed: (1) a standardised interview focusing on ocular and oral sicca symptoms; ${ }^{6}(2)$ physical examination; (3) tests for objective ocular involvement 
Clinical data of patients with an abnormal sublabial salivary gland biopsy specimen

\begin{tabular}{lllllllll}
\hline $\begin{array}{l}\text { Patient } \\
\text { No }\end{array}$ & Age/sex & Neuropathy & $\begin{array}{l}\text { Ocular } \\
\text { symptoms }\end{array}$ & $\begin{array}{l}\text { Oral } \\
\text { symptoms }\end{array}$ & $\begin{array}{l}\text { Schirmer } \\
\text { OD/OS }\end{array}$ & TLC & ESR & Serology \\
\hline 1 & $70 / \mathrm{F}$ & SM & No & Yes & $16 / 15$ & 4800 & 27 & $\begin{array}{l}\text { Anti-Ro/SSA } \\
\gamma \text {-Globuline }\end{array}$ \\
2 & & & & & & & & \\
3 & T9/F & SM & Yes & No & $2 / 7$ & 2200 & 19 & $\begin{array}{l}\gamma \text {-Globuline } \\
\gamma \text {-Globuline }\end{array}$ \\
\hline
\end{tabular}

$\mathrm{S}=$ Sensory $\mathrm{SM}=$ sensorimotor $\mathrm{OD}=$ right eye OS = left eye TLC = mean tear lysozyme concentration of both eyes $(\mu \mathrm{g} / \mathrm{ml}) ; \mathrm{ESR}=$ erythrocyte sedimentation rate $(\mathrm{mm} / \mathrm{h})$.

by means of an unanesthetised Schirmer's test and tear fluid lysozyme concentration or Rose bengal staining score; ${ }^{6}(4)$ additional laboratory investigations (repeated erythrocyte sedimentation rate, serum agar gel electrophoresis, quantitative immunoglobulin assessment, repeated antinuclear antibodies, anti-Ro/SSA and anti-La/SSB autoantibodies, and rheumatoid factor); (5) SSG biopsy for histopathological examination, according to Greenspan et al. ${ }^{7}$ We included the method described by Bodeutsch et al to improve the sensitivity and specificity of the diagnosis of Sjögren's syndrome in SSG biopsy specimens. ${ }^{8}$ They determined quantitative immunohistological data, based on the percentages of $\operatorname{IgA}, \operatorname{IgG}$, and IgM containing plasma cells in the SSG biopsy specimen. Using these quantitative immunohistological criteria (\% IgA producing plasmacells < 70), sensitivity, and specificity of SSG biopsy in the diagnosis, Sjögren's syndrome is at least $95 \%$.

As histopathology of labial salivary glands has long been considered the most disease specific and sensitive single test in the diagnosis of Sjögren's syndrome, we classified patients as having Sjögren's syndrome on the basis of an SSG biopsy specimen compatible with Sjögren's syndrome and positive results on at least two of the following tests: (a) ocular symptoms (existing for more than three months), $(b)$ oral

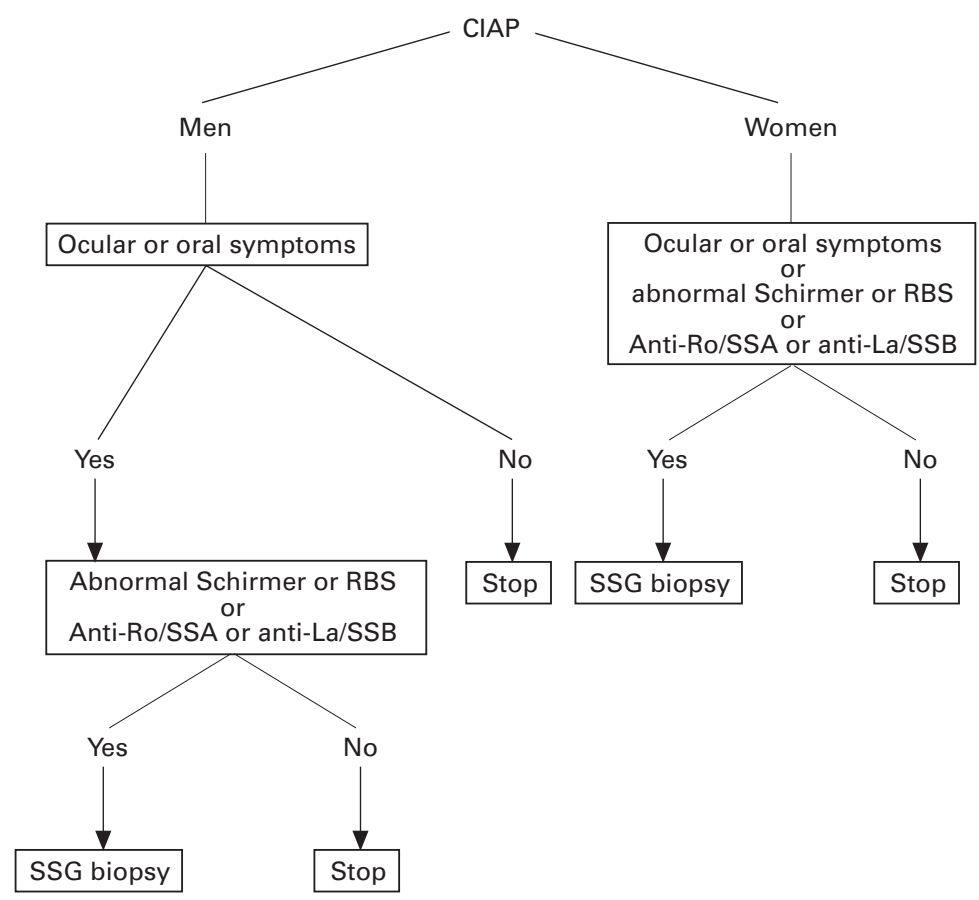

Figure 1 Diagnostic approach with respect to Sjögren's syndrome in patients with chronic idiopathic axonal polyneuropathy $(C I A P)$. SSG= sublabial salivary gland biopsy. symptoms (existing for more than three months), (c) objective ocular signs, and (d) the presence of autoantibodies: anti-Ro/SSA, anti$\mathrm{La} / \mathrm{SSB}$, or rheumatoid factor.

\section{Results}

Sixty five patients were studied ( $32 \mathrm{men}$ ). The mean age was 63 (range 47-79) years and on clinical grounds, 41 patients had a pure sensory and 24 a sensorimotor neuropathy. On entry into the study, the mean duration of the disease was 5.1 (SD 3.4) years. The clinical course of the neuropathy had been slowly progressive or stable. Informed consent for an SSG biopsy was obtained in 49 patients.

In three women, the SSG biopsy specimens were diagnostic for Sjögren's syndrome. More detailed results of diagnostic tests that led to a diagnosis of primary Sjögren's syndrome in these three patients are given in the table. Patient 1 had a sensorimotor neuropathy, which started with paraesthesia in the feet 10 years before study entry. Sural nerve biopsy showed a decreased number of large myelinated nerve fibres and features of axonal degeneration, but no signs of vasculitis or inflammatory disease. Besides the abnormal SSG biopsy $(<70 \%$ IgA producing plasma cells), she had oral symptoms and also anti-Ro/ SSA autoantibodies. One year after study entry, the symptoms and signs of Siögren's syndrome became more clear, as she developed bilateral parotid gland swelling, ocular symptoms, and an abnormal Schirmer's test. Patient 2 also had a sensorimotor neuropathy. Her symptoms started four years before study entry with walking difficulties caused by weakness of peroneal and anterior tibial muscles. Besides an abnormal SSG biopsy ( $<70 \%$ IgA producing plasma cells and a focus score $>1$ ), she had ocular symptoms and an abnormal Schirmer's test. The serum $\gamma$-globulin content was raised, but autoantibodies were absent. On clinical follow up, the neuropathy was progressive, leading to disabling weakness of distal leg muscles and severe distal sensory disturbances. She was treated with prednisone $(1 \mathrm{mg} / \mathrm{kg})$ and the progression of the neuropathy stopped. Patient 3 had a pure sensory neuropathy that started with paraesthesia and numbness in the feet four years before study entry. Her SSG biopsy specimen showed $<70 \%$ IgA producing plasma cells and a focus score $>1$. She had ocular symptoms, an abnormal Schirmer's test, abnormal tear lysozyme concentration, and abnormal Rose bengal staining score. During follow up, the course of the neuropathy remained stable and purely sensory.

In 46 patients with normal SSG biopsy findings, abnormalities on the other tests for Sjögren's syndrome were sometimes noticed. Five patients had ocular symptoms, four had oral symptoms, and an abnormal Schirmer's test was found in eight patients. More than one abnormal test result was found in two of eight patients with an abnormal Schirmer's test, who also had ocular or oral symptoms and in one patient with an abnormal Schirmer's test who also had a reduced tear lysozyme concentration. In these 46 patients with normal SSG 
biopsy findings, SSA or SSB autoantibodies were absent. One woman had antinuclear antibodies but did not have any other feature of generalised autoimmune disease. After two years of follow up, she still has positive antinuclear antibodies as the only abnormal finding.

In 16 patients who did not allow a SSG biopsy, one had ocular and oral symptoms, two had ocular or oral symptoms, and another two an abnormal Schirmer's test as the only abnormal finding.

\section{Discussion}

The results of this study indicate that the diagnosis of Sjögren's syndrome should be considered in patients with CIAP, as three of 49 patients who underwent SSG biopsy had definite primary Sjögren's syndrome. None of these patients complained spontaneously about sicca symptoms and their clinical neurological picture did not differ from the other patients in the study. In patients with Sjögren's syndrome and distal symmetric neuropathy, neurological involvement is often the presenting manifestation and the main feature of the disease, whereas an onset with sicca symptoms or systemic involvement is less common. ${ }^{3}$ Furthermore, patients with Sjögren's syndrome and distal symmetric polyneuropathy may develop Sjögren's syndrome at a significantly older age than patients with Sjögren's syndrome without neuropathy. ${ }^{2}$ It can therefore be hypothesised that patients with Sjögren's syndrome and distal axonal neuropathy represent a subset of patients with Sjögren's syndrome. Gemignani et al state that aging is a critical factor for the development of this kind of neuropathy in Sjögren's syndrome, possibly favouring microangiopathic changes. ${ }^{2}$ On the other hand, clear signs of vasculitis are sometimes present in nerve biopsies of patients with Sjögren's syndrome and neuropathy, but in these cases the neuropathy usually has the character of a mononeuropathy multiplex; this manifestation occurs mostly after the diagnosis has been made and when other (systemic) complications of Sjögren's syndrome are also present. $^{4}$

It is important to notice that none of the individual tests is useful as a screening method for Sjögren's syndrome, due to low sensitivity and specificity of these tests when performed alone, ${ }^{6}$ which was also illustrated in the underlying study. We used classification criteria for Sjögren's syndrome adapted from the European Community Study Group on diagnostic criteria for Sjögren's syndrome, in which a diagnosis is made when a positive result on four of six tests is present. ${ }^{6}$ Furthermore, as the SSG biopsy is considered the most reliable single test in terms of both sensitivity and specificity, ${ }^{6}$ an abnormal SSG biopsy was required in our study for the diagnosis of Sjögren's syndrome.

As in this study, most patients with Sjögren's syndrome have a raised erythrocyte sedimentation rate. ${ }^{4}$ However, a normal erythrocyte sedimentation rate does not exclude the presence of Sjögren's syndrome ${ }^{4}$ and therefore cannot be used as a deciding factor in the diagnostic approach in patients with CIAP. The fact that in this study only women fulfilled the Sjögren's syndrome criteria could be coincidental, although the a priori chance of primary Sjögren's syndrome in women is much higher than in men (9:1). ${ }^{4}$ Taking into account the costs, inconvenience, and morbidity (SSG biopsy) of extensive testing for Sjögren's syndrome and the very low a priori chance of primary Sjögren's syndrome in men, we suggest performing further tests for the syndrome in men with CIAP only when ocular or oral sicca symptoms are present (figure). However, in women with CIAP we suggest a full investigation for Sjögren's syndrome that includes a questionnaire for ocular and oral symptoms, Schirmer's test, or Rose bengal staining score and determination of anti-Ro/SSA and anti-La/ SSB autoantibodies (figure). When an abnormal test result is found in any of these tests, an SSG biopsy should be performed in women to see if the diagnosis of Sjögren's syndrome can be made.

We are grateful to Dr PCM de Wilde and Dr ThM Vroom, for reviewing the labial salivary gland biopsy specimens.

1 Mc Leod JG, Tuck RR, Pollard JD, Cameron J, Walsh JC Chronic polyneuropathy of undetermined cause. 7 Neurol Neurosurg Psychiatry 1984;47:530-5.

2 Gemignani F, Marbini A, Pavesi G, et al. Peripheral neuropathy associated with primary Sjögren's syndrome. $\mathcal{F}$ neuropathy associated with primary Sjogren's

3 Mellgren SI, Conn DL Clarke Stevens J, et al. Peripheral neuropathy in primary Sjögren's syndrome. Neurology 1989;39:390-4.

4 Kruize AA, Hene RJ, Van der Heide A, et al. Long-term follow up of patients with Sjögren's syndrome. Arthritis Rheum 1996;39:297-303.

5 Notermans NC, Wokke JHJ, Fransen H, et al. Chronic idiopathic polyneuropathy presenting in middle or old age: clinical and electrophysiological study of 75 patients. $\mathscr{f}$ Neurol Neurosurg Psychiatry 1993;56:1066-71.

6 Vitali C, Bombardieri S, Moutsopoulos HM, et al. Assessment of the European classification criteria for Sjögren's syndrome in a series of clinically defined cases: results of a prospective multicenter study. Ann Rheum Dis 1996;55:116-21.

7 Greenspan JS, Daniels TE, Talal N, Sylvester RA. The histopathology of Sjögren's syndrome in labial salivary gland biopsies. Oral Surg 1974;37:217-99.

8 Bodeutsch C, De Wilde PCM, Kater L, et al. Quantitative immunohistologic criteria are superior to the lymphocytic focus score criterion for the diagnosis of Sjögren's syndrome. Arthritis Rheum 1992;35:1075-87. 